\title{
What will it take to implement genomics in practice? Lessons from the IGNITE Network
}

\author{
Geoffrey S Ginsburg*,1, Carol R Horowitz ${ }^{2} \&$ Lori A Orlando 1 \\ ${ }^{1}$ Center for Applied Genomics \& Precision Medicine, Duke University, Durham, NC, USA \\ ${ }^{2}$ Department of Population Health Science and Policy Icahn School of Medicine at Mount Sinai, NY, USA \\ *Author for correspondence: Tel.: + 1919668 6210; Geoffrey.ginsburg@duke.edu
}

\begin{abstract}
"IGNITE provides an exemplar of this novel science and it has sparked an opportunity for community-based practices to begin implementing genomics in their own environments and for patients and advocates to form genomic programs designed to improve their own health."
\end{abstract}

First draft submitted: 20 February 2019; Accepted for publication: 4 April 2019; Published online: 23 July 2019

Keywords: clinical genomics $\bullet$ genomic medicine $\bullet$ implementation

Capitalizing on its successful track record using DNA technologies to define variants underpinning disease and to enable the sequencing of the human genome, the National Human Genome Research Institute (NHGRI) is shifting its focus toward advancing the science of medicine and delivery of healthcare through its genomic medicine demonstration projects $[1,2]$. These projects harness implementation science, the study of methods to promote the integration of research findings and evidence into healthcare practice, as a key step in the diffusion of innovation and its broad adoption by the practicing community. The recently created Implementing Genomics in Practice (IGNITE) network is the leading edge of genomic medicine implementation in USA [3]. It is nimble and willing to share information in real time, helps stakeholders in genomic medicine research improve their ability to implement and recruit and is creating the scientific foundation for the future of genomic medicine implementation. While many aspects of implementation science are generic, the unique features of genomic medicine call for adapting current implementation science models to meet the needs of patients, providers and health systems adopting these complex interventions. Since 2013, the network has faced numerous challenges. To enable future research, those challenges and our insights for solutions are highlighted below:

\section{Genomic medicine implementation requires a transdisciplinary team}

Most genomics researchers are not implementation scientists. Thus, the first step in enabling genomic medicine implementation is to assemble teams with the right expertise. At minimum, these should include genomic medicine researchers, implementation scientists, health services and outcomes researchers, patients from diverse demographic groups and members of the care delivery teams where implementation is to occur.

\section{Developing an implementation framework}

Implementation frameworks can guide intervention deployment, assessment and analyses. Without a framework, implementation is likely to be unstructured and haphazard. This could widen rather than close knowledge gaps, cause evaluations to omit key domains and different teams to use different metrics, thwarting cross-site and crossnetwork comparisons. For example, if the implementation climate and perceived value of a genomics intervention are critical elements of intervention success, but are not measured, investigators may be unable to explain why deployment was successful in some settings, but not others. IGNITE adopted and adapted the consolidated framework for implementation research (CFIR) in creating a network focused on developing lessons for the larger community. With no well-developed models specific to genomic medicine, IGNITE successfully employed CFIR, refining it to create a draft genomic medicine implementation research model [4] that can help investigators understand, standardize and collect key implementation aspects necessary to generalize findings for uptake in the 'real world'. 


\section{Determining the right design for an implementation study}

Implementation study design is determined by the readiness of the genomic medicine technology for use in clinical care, and by how prepared the environment is to receive it. Given the limitations of the current evidence base for genomic medicine interventions, most IGNITE projects (e.g., pharmacogenetics or disease susceptibility) focused primarily on assessing clinical effectiveness outcomes, while gathering information relevant to the delivery of the intervention' to inform potential for future, real-world implementation. This is called a 'hybrid' type I clinical-effectiveness trial, since data on the effectiveness and delivery are collected simultaneously. When evidence for the intervention is generally accepted by the clinical community (e.g., family history), researchers prioritize implementation outcomes in order to enable its widespread use, but also gather data on effectiveness. This is known as a hybrid type III clinical effectiveness trial [5]. The choice of approach varies depending on how much is known about the intervention and the setting; but hybrid designs are clearly an important step as they link clinical outcomes to organizational characteristics, informing efforts at optimizing both. They also dictate the type and prioritization of measures to collect, as well as the types of stakeholders to engage.

\section{Optimizing the setting \& personnel to carry out the implementation research in the clinic}

Genomic medicine investigators need to embrace implementation science, and front-line clinicians, staff and patients will also need to embrace translational genomics. Pre-implementation research is often overlooked as a critical element to ensure that researchers understand and take into account the priorities, concerns and educational needs of these key stakeholders before implementation begins. In IGNITE, the impact of genomic medicine interventions on clinical workflow were often underappreciated, as were concerns of providers new to genomics research, and even research in general. Local clinician and patient champions for genomic medicine can be key in garnering support for studies, especially in practices that care for low-income and racial-ethnic minority patients who are often the last to benefit from these advances. Creating an investigator-clinical champion pairing can enhance success with the former keeping the latter updated on the research and stimulating excitement for the project, and the latter providing continuous feedback on what is working and what is not so that researchers can optimize implementations to fit the 'real world'.

\section{Genomic medicine research is information technology (IT) intensive}

Given the nature of genomic information, most IGNITE studies incorporated genomic data into an electronic health record (EHR) or extracted phenotypic data out of it. Broad implementation will require that IT solutions work with any EHR. Some IGNITE projects used FHIR as a platform to achieve this with success. In addition, IGNITE was launched in the era of EHR adoption and adaptation. Several sites underwent EHR vendor changes mid-stream, necessitating major changes in research data flow and disruption of trial recruitment. While major transitions of EMR systems should soon stabilize, these systems are continually evolving, and IT challenges are likely to continue for the foreseeable future. The critical role of IT in streamlining workflow means that genomics will more effectively enter clinical environments if IT leadership at the implementing institution prioritizes its incorporation and local clinicians and health system leaders sanction novel genomic medicine interventions.

\section{Genomic medicine research is dynamic}

Since IGNITE was first conceived, there has been an evolution of the science of genomics and the evidence base for many tests and technologies. Other NHGRI sponsored networks have progressed the evidence base for sequencing of adults and newborns, rare disease diagnosis and EHR-enabled pharmacogenetics, and the work is ongoing. To facilitate knowledge translation for early (and late) adopters, IGNITE created a knowledge hub, the IGNITE SPARK toolbox [6], to establish and maintain a standard for genomic medicine implementation and to disseminate this information to the clinical community.

\section{The need for genomic medicine implementation continues}

The solicitation for IGNITE II was announced last with a distinct focus on building the evidence for clinical utility of genomic medicine in diverse, underserved and underrepresented minority populations and the health systems that serve them [7]. The USA, like many other nations, has a very heterogeneous healthcare delivery system with large variations in practice patterns, access to genomic technology and literacy of patients and providers. Carrying out this strategic research agenda will require an investigator community that is prepared to move beyond the traditional research sites afforded by academic and tertiary care centers to the less charted areas of community-based 
practices. While this transition may be challenging, it is critical for the health of the population to bring genomic medicine from the ivory tower to the general population.

Research on the implementation of genomics into medicine is in many ways a disruptive exercise, bringing together people who have not historically partnered extensively, to develop and use new methods and approaches. IGNITE provides an exemplar of this novel science and it has sparked an opportunity for community-based practices to begin implementing genomics in their own environments and for patients and advocates to form genomic programs designed to improve their own health. These stakeholders will be part of an ever-growing network that continuously learns, refining how to implement genomics and other novel technologies for the benefit of all.

\section{Acknowlegments}

Investigators who contributed from the IGNITE Network are: J Johnston, University of Florida; T Pollin, University of Maryland; J Denny and M Levy, Vanderbilt University; T Skaar and P Dexter, Indiana University; CR Horowitz, Icahn School of Medicine at Mount Sinai; E Madden and T Manolio, National Human Genome Research Institute; GS Ginsburg and L Orlando, Duke University.

\section{Financial \& competing interests disclosure}

This work was supported by grants from the NIH (U01 HG007269, U01 HG007253, U01 HG007762, U01 HG007282, U01 HG007775, U01 HG007278 and by the NIH IGNITE Network (http://ignite-genomics.org/). The authors have no other relevant affiliations or financial involvement with any organization or entity with a financial interest in or financial conflict with the subject matter or materials discussed in the manuscript apart from those disclosed.

No writing assistance was utilized in the production of this manuscript.

\section{References}

1. Green ED, Guyer MS. National Human Genome Research Institute. Charting a course for genomic medicine from base pairs to bedside. Nature 470(7333), 204-213 (2011).

2. NIH. https://grants.nih.gov/grants/guide/rfa-files/RFA-HG-12-006.html

3. Weitzel KW, Alexander M, Bernhardt BA et al. The IGNITE network: a model for genomic medicine implementation and research. BMC Med. Genomics. 9(1) doi: 10.1186/s12920-015-0162-5 (2016).

4. Orlando LA, Sperber NR, Voils C et al. Developing a common framework for evaluating the implementation of genomic medicine interventions in clinical care: the IGNITE Network's Common Measures Working Group. Genet. Med. 20(6), 655-663 (2017).

5. Curran GM, Bauer M, Mittman B, Pyne JM, Stetler C. Effectiveness-implementation hybrid designs: combining elements of clinical effectiveness and implementation research to enhance public health impact. Med. Care 50(3), 217-226 (2012).

6. Ignite. https://ignite-genomics.org/spark-toolbox/

7. NIH. https:/grants.nih.gov/grants/guide/rfa-files/RFA-HG-17-010.html 